\title{
A new perspective on paranormed Riesz sequence space of non-absolute type
}

\author{
Murat Candan \\ Department of Mathematics, Faculty of Arts and Sciences, Inönü University, Malatya City, 44280, TURKEY \\ Email: murat.candan@inonu.edu.tr
}

Copyright (C)2015 Murat Candan. This is an open access article distributed under the Creative Commons Attribution License, which permits unrestricted use, distribution, and reproduction in any medium, provided the original work is properly cited.

\begin{abstract}
The current article mainly dwells on introducing Riesz sequence space $r^{q}\left(\widetilde{B}_{u}^{p}\right)$ which generalized the prior studies of Candan \& Güneş [28], Candan \& Kılınç [30] and consists of all sequences whose $R_{u}^{q} \widetilde{B}$-transforms are in the space $\ell(p)$, where $\widetilde{B}=B\left(r_{n}, s_{n}\right)$ stands for double sequential band matrix $\left(r_{n}\right)_{n=0}^{\infty}$ and $\left(s_{n}\right)_{n=0}^{\infty}$ are given convergent sequences of positive real numbers. Some topological properties of the new brand sequence space have been investigated as well as $\alpha$ - $\beta$ - and $\gamma$-duals. Additionally, we have also constructed the basis of $r^{q}\left(\widetilde{B}_{u}^{p}\right)$. Eventually, we characterize a matrix class on the sequence space. These results are more general and more comprehensive than the corresponding results in the literature.
\end{abstract}

Keywords: Paranormed sequence space, alpha-, beta- and gamma-duals and matrix mappings.

\section{Introduction}

First of all, we are going to introduce some notations and definitions which are going to be needed in later sections of the article. Let us begin by discussing the concept of a sequence. There are many ways of defining a sequence, each of which is an equivalent way of defining the same thing. A sequence is simply an ordered list $x_{1}, x_{2}, \ldots, x_{n}, \ldots$ of numbers. Such a sequence is called an infinite sequence. In this article sequences will be infinite thus so from now on when we speak of sequences we will mean infinite sequences. A slightly more sophisticated way of representing the sequence $a_{1}, a_{2}, \ldots, a_{n}, \ldots$ is with the notation $\left\{a_{i}\right\}_{i=0}^{\infty}$. We will often abbreviate $\left\{a_{i}\right\}_{i=0}^{\infty}$ simply with $\left\{a_{i}\right\}$. A sequence $\left\{a_{n}\right\}$ converges with limit $a$ if each neighborhood of $a$ contain almost all terms of the sequence. In this case we say that $\left\{a_{n}\right\}$ converges (or convergent) to $a$ as $n$ goes to $\infty$. In a common parlance the words series and sequence are essentially synonomous, however, in mathematics the distinction between two is that a series is the sum of the terms of a sequence. Let $\left\{a_{n}\right\}$ be a sequence and define a new sequence $\left\{s_{n}\right\}$ by the recursion relation $\left\{s_{1}\right\}=\left\{a_{1}\right\}$ and $\left\{s_{n+1}\right\}=\left\{s_{n}\right\}+\left\{a_{n+1}\right\}$. The sequence $\left\{s_{n}\right\}$ is called the sequence of partial sums of $\left\{a_{n}\right\}$. Let $\left\{s_{n}\right\}$ be sequence of partial sums of $\left\{a_{n}\right\}$. If $\left\{s_{n}\right\}$ converges we say that $\left\{a_{n}\right\}$ is summable. In this case, we denote the $\lim _{k \rightarrow \infty} s_{n}$ by $\sum_{j=0}^{\infty} a_{j}$. The expression $\sum_{j=0}^{\infty} a_{j}$ is called an infinite series whether or not the sequence $\left\{a_{n}\right\}$ is summable. When we are given an infinite series $\sum_{j=0}^{\infty} a_{j}$ the sequence $\left\{a_{n}\right\}$ is called the sequence of terms. If the sequence of terms is summable, the infinite series is said to be convergent. The set of all convergent sequences in $\mathbb{K}$ are denoted by $c$. A sequence $\left\{a_{n}\right\}$ in $\mathbb{K}$ is called a null sequence if it converges to zero. The set of all null sequences in $\mathbb{K}$ are denoted by $c_{0}$, where $\mathbb{K}$ denotes either of fields $\mathbb{R}$ and $\mathbb{C}$. A sequence is bounded if the set of its terms have an upper bound and a lower bound. The set of all bounded sequences is denoted by $\ell_{\infty}$. Any vector subspace 
of $\omega=\omega(\mathbb{K})=\mathbb{K}^{\mathbb{N}}$ is known as a sequence space and $\mathbb{N}:=\{0,1,2, \ldots\}$. It is clear that the sets $c, c_{0}$ and $\ell_{\infty}$ are the subspaces of the $\omega$. Therefore, $c, c_{0}$ and $\ell_{\infty}$, equipped with a vector space structure, forms a sequence space. Also by $b s, c s, \ell_{1}$ and $\ell_{p}$ we denote the spaces of all bounded, convergent, absolutely and $p$-absolutely convergent series, respectively.

Now, let us give the definition of the triangle matrix. Let $T=\left(t_{n k}\right)$ be a triangle matrix, that is $t_{n k}=0$ for $k>n$ and $t_{n n} \neq 0$ for all $n \in \mathbb{N}$. It is clear that $A(B x)=(A B) x$ holds for the triangle matrices $A, B$ and a sequence $x$. Moreover, a triangle matrix $U$ uniquely has an inverse $U^{-1}=V$ which is also a triangle matrix. Thus, $x=U(V x)=V(U x)$ holds for all $x \in \omega$.

The concept of matrix domain plays one of the most important role in this article. Therefore, its definition is presented in this paragraph. The domain $\lambda_{A}$ of an infinite matrix $A$ in a sequence space $\lambda$ is defined by

$\lambda_{A}:=\left\{x=\left(x_{k}\right) \in \omega: A x \in \lambda\right\}$,

which is a sequence space.

Even though generally the new sequence space $\lambda_{A}$ produced by the limitation matrix $A$ using a sequence space $\lambda$ is either the expansion or the contraction of the space $\lambda$ itself, sometimes those spaces may be observed overlap. In fact, it is easy see that the inclusion $\lambda_{S} \subset \lambda$ strictly holds for $\lambda \in\left\{\ell_{\infty}, c, c_{0}\right\}$. Because of this property, it can be deduced that the inclusion $\lambda \subset \lambda_{\Delta}$ (1) also strictly holds for $\lambda \in\left\{\ell_{\infty}, c, c_{0}, \ell_{p}\right\}$. But, when $\lambda:=c_{0} \oplus \operatorname{span}\{z\}$ is defined with $z=\left((-1)^{k}\right)$, that is $x \in \lambda$ iff $x:=s+\alpha z$ for some $s \in c_{0}$ and some $\alpha \in \mathbb{C}$, and take the matrix $A$ with the rows $A_{n}$ described by $A_{n}:=(-1)^{n} e^{(n)}$ for all $n \in \mathbb{N}$ into consideration, we obtain $A e=z \in \lambda$ while $A z=e \notin \lambda$ resulting in the sequences $z \in \lambda \backslash \lambda_{A}$ and $e \in \lambda_{A} \backslash \lambda$, here $e=(1,1,1, \ldots)$ and $e^{(n)}$ represents a sequence of which only non-zero element is a 1 on the $n^{\text {th }}$ place for each $n \in \mathbb{N}$. In other words, the sequence spaces $\lambda_{A}$ and $\lambda$ overlap while none of them contains the other one. For more detail on the domains of some triangle matrices in certain sequence spaces, the reader may refer to Baar's book entitled "Summability Theory and Its Applications" (see [1, p. 50]).

Another essential definition is the paranorm definition which is going to be needed in later. Now, let us give this concept. The function $g$ on a $X$ satisfies the properties of a paranorm $g(\theta)=0, g(x)=g(-x), g(x+y)=g(x)+g(y)$, $\left|\alpha_{n}-\alpha\right| \rightarrow 0$ and $g\left(x_{n}-x\right) \rightarrow 0$ imply $g\left(\alpha_{n} x_{n}-\alpha x\right) \rightarrow 0$ for all $\alpha \in \mathbb{R}$ and all $x \in X$, where $\theta$ is the zero vector in the linear space $X$. Recall that a linear topological space $X$ over the real field $\mathbb{R}$ with a paranorm obeying these rules is called a paranormed space.

From now on, let us assume that $\left(p_{k}\right)$ be a bounded sequence of strictly positive real numbers with sup $p_{k}=H$ and $M=\max \{1, H\}$ and $1 / p_{k}+1 / p_{k}^{\prime}=1$ provided $1<i n f p_{k} \leq H<\infty$. Then, the linear spaces $\ell_{\infty}(p)$ and $\ell(p)$ were defined by Maddox in [2] and [3] (see also Simons [4] and Nakano [5]) as follows:

$\ell(p)=\left\{x=\left(x_{k}\right) \in w: \sum_{k}\left|x_{k}\right|^{p_{k}}<\infty\right\}$

and

$\ell_{\infty}(p)=\left\{x=\left(x_{k}\right) \in w: \sup _{k \in \mathbb{N}}\left|x_{k}\right|^{p_{k}}<\infty\right\}$,

which are the complete spaces paranormed by

$g_{1}(x)=\left(\sum_{k}\left|x_{k}\right|^{p_{k}}\right)^{1 / M}$ and $g_{2}(x)=\sup _{k \in \mathbb{N}}\left|x_{k}\right|^{p_{k} / M}$ iff inf $p_{k}>0$

, respectively.

For the sake of simplicity, here and in what follows, it will be assumed that the summation without limits runs from 0 to $\infty$.

Recently, the approach to construct a new sequence space by means of the matrix domain of a particular triangle has been used by some of the writers in many research articles. They defined and examined the sequence spaces $X_{p}=\left(\ell_{p}\right)_{C_{1}}$ in [6], $r^{t}(p)=(\ell(p))_{R_{t}}$ in [7], $e_{p}^{r}=\left(\ell_{p}\right)_{E^{r}}$ and $e^{r}(p)=(\ell(p))_{E^{r}}$ in $[8,9,10] . Z\left(u, v, \ell_{p}\right)=\left(\ell_{p}\right)_{G(u, v)}$ and $\ell(u, v, p)=(\ell(p))_{G(u, v)}$ in $[11,12], a^{r}(p)=\left(\ell_{p}\right)_{A^{r}}$ and $a^{r}(u, p)=(\ell(p))_{A_{u}^{r}}$ in $[13,14], b v_{p}=\left(\ell_{p}\right)_{\Delta}$ and $b v(u, p)=(\ell(p))_{A_{u}}$ in $[15,16,17], \overline{\ell(p)}=(\ell(p))_{S}$ in [18], $\ell_{p}^{\lambda}=\left(\ell_{p}\right)_{\Lambda}$ in [19], $\lambda_{B(r, s)}$ in [20] $\lambda_{B(\tilde{r}, \tilde{s})}$ in [21], $f_{0}(B)$ and $f(B)$ in [22], $f_{0}(\widetilde{B})$ and $f(\widetilde{B})$ in [23] and etc., where $C_{1}=\left\{c_{n k}\right\}, \quad R^{t}=\left\{r_{n k}^{t}\right\}, \quad E^{r}=\left\{e_{n k}^{r}\right\}, \quad S=\left\{s_{n k}\right\}, \quad \Delta=$ $\left\{\delta_{n k}\right\}, \quad G(u, v)=\left\{g_{n k}\right\}, \quad \Delta^{(m)}=\left\{\Delta_{n k}^{(m)}\right\}, \quad A^{r}=\left\{a_{n k}^{r}\right\}, \quad A_{u}^{r}=\left\{a_{n k}(r)\right\}, \quad A^{u}=\left\{a_{n k}^{u}\right\}, \quad B(r, s)=$ 
$\left\{b_{n k}(r, s)\right\}, \quad B(\tilde{r}, \tilde{s})=\left\{b_{n k}(\tilde{r}, \tilde{s})\right\}, \quad \Lambda=\left\{\lambda_{n k}\right\}_{n, k=0}^{\infty}$ and $A(\lambda)=\left\{a_{n k}(\lambda)\right\}$ denote the Cesro, Riesz, Euler, generalized weighted means or factorable matrix, summation matrix, difference matrix, generalized difference matrix and sequential band matrix, respectively. Moreover, the ones who are more interested in the subject are advised to $\operatorname{read}[24,25,26,27,28,29,30,31,32,33,34,35,36,37,38,39,40,41,42,43,44,45,46,47,48,49,50,51,52]$. We should note here, there are many different ways to construct new sequence spaces from old ones. To get more detailed information, one can look at the articles [53, 54, 55, 56].

In this paragraph, we shall introduce the notion of a matrix transformation from $X$ to $Y$. Let $X, Y$ be any two sequence spaces. Given any infinite matrix $A=\left(a_{n k}\right)$ of real numbers $a_{n k}$, where $n, k \in \mathbb{N}$, any sequence $x$, we write $A x=\left((A x)_{n}\right)$, the $A$-transform of $x$, if $(A x)_{n}=\sum_{k} a_{n k} x_{k}$ converges for each $n \in \mathbb{N}$. If $x \in X$ implies that $A x \in Y$ then we say that $A$ defines a matrix mapping from $X$ into $Y$ and denote it by $A: X \rightarrow Y$. By $(X: Y)$, we mean the class of all infinite matrices such that $A: X \rightarrow Y$.

In the present article, we introduce the new sequence spaces derived by Riesz mean $\left(R, q_{n}\right)$ and generalized difference matrix $\widetilde{B}(r, s)$.

The layout of the rest of the present article is organized as follows:

Section 2 is devoted to the spaces of difference sequences and some historical developments related to this matter are given. Additionally, the concept of generalized difference matrix is introduced. In section 3 , the paranormed sequence space $r^{q}\left(\widetilde{B}_{u}^{p}\right)$ of non-absolute type which is the set of all sequences whose $R_{u}^{q} \widetilde{B}$-transforms are in the spaces $\ell(p)$ and then their alpha-, beta- and gamma-duals are computed. In addition to this, the basis of the space $r^{q}\left(\widetilde{B}_{u}^{p}\right)$ is obtained. In the final section of the article, we characterize a matrix class on the sequence space.

\section{Difference sequence spaces}

In this section, we are going to give some knowledge about literature concerning the spaces of difference sequence.

The difference sequence spaces have been studied by several authors in different ways. At first, Kizmaz introduced the difference sequence space in 1981. Now, let us briefly explain further. If $\lambda \in\left\{\ell_{\infty}, c, c_{0}\right\}$ then, $\lambda(\Delta)$ consisting of the sequences $x=\left(x_{k}\right)$ such that $\left(x_{k}-x_{k+1}\right) \in \lambda$ is called as the difference sequence spaces which were introduced by Kizmaz [57]. In recent years, the difference spaces $b v_{p}$ consisting of the sequences $x=\left(x_{k}\right)$ such that $\left(x_{k}-x_{k-1}\right) \in \ell_{p}$ have been studied in the case $0<p<1$ by Altay and Başar [58], and in the case $1 \leq p<\infty$ by Başar and Altay [59], and Çolak, Et and Malkowsky [60].

The concept of difference sequences was generalized by Çolak and Et [61]. They defined and examined the sequence spaces

$\Delta^{m} \lambda=\left\{x=\left(x_{k}\right) \in \omega: \Delta^{m} x \in \lambda\right\}$

where $\Delta^{1} x=\left(x_{k}-x_{k+1}\right)$ and $\Delta^{m} x=\Delta\left(\Delta^{m-1} x\right)$ for $m \in\{1,2,3, \ldots\}$. In [62], Malkowsky and Parashar introduced the sequence spaces as follows

$\Delta^{(m)} \lambda=\left\{x=\left(x_{k}\right) \in \omega: \Delta^{(m)} x \in \lambda\right\}$,

where $m \in \mathbb{N}, \Delta^{(1)} x=\left(x_{k}-x_{k-1}\right)$ and $\Delta^{(m)} x=\Delta^{(1)}\left(\Delta^{(m-1)} x\right)$. More recently, in [63], Polat and Başar introduced the spaces $e_{0}^{r}\left(\Delta^{(m)}\right), e_{c}^{r}\left(\Delta^{(m)}\right)$ and $e_{\infty}^{r}\left(\Delta^{(m)}\right)$ consisting of all sequences whose $m^{t h}$ order differences are in the Euler spaces $e_{0}^{r}, e_{c}^{r}$ and $e_{\infty}^{r}$, respectively. Finally, Altay [64] studied the space $\ell_{p}\left(\Delta^{(m)}\right)$ consisting of all sequences whose $m^{\text {th }}$ order differences are $p$-absolutely summable which is a generalization of the spaces $b v_{p}$ defined by Başar and Altay [59], and Çolak, Et and Malkowsky [60].

Let $r_{n}$ and $s_{n}$ be non-zero real numbers for all $n \in \mathbb{N}$, and define the double sequential band matrix $\widetilde{B}=$ $B(\widetilde{r}, \widetilde{s})=\left\{b_{n k}(\widetilde{r}, \widetilde{s})\right\}$ by

$b_{n k}(\widetilde{r}, \widetilde{s}):=\left\{\begin{array}{cll}r_{n} \quad, \quad(k=n), \\ s_{n}, \quad(k=n-1), \\ 0 \quad, \quad(0 \leq k<n-1 \quad \text { or } k>n),\end{array}\right.$

for all $k, n \in \mathbb{N}$. Let us note here that the matrix $B(\widetilde{r}, \widetilde{s})$ can be reduced to the generalized difference matrix $B(r, s)$ in the case $r_{n}=r, s_{n}=s$ for all $n \in \mathbb{N}$. Thus, the results related to the domain of the matrix $B(\widetilde{r}, \widetilde{s})$ are the generalization of the corresponding consequences of the matrix domain of $B(r, s)$.

Generalized difference matrix $B(r, s)$ has been used by some of the writers in many research articles. Purely for the development of this approach, the articles Kiriş̧̧i and Başar [20, 22] are recommended. 


\section{The Riesz Sequence Space $r^{q}\left(\widetilde{B}_{u}^{p}\right)$ of Non-absolute Type}

In this section, we will focus on the new paranormed sequence space $r^{q}\left(\widetilde{B}_{u}^{p}\right)$, using the Riesz mean and double sequential band matrix.

Before going into the details, we first introduce some notations and definitions. We begin by talking about the Riesz mean.

The transformation given by

$t_{n}=\frac{q_{0} x_{0}+q_{1} x_{1}+\cdots+q_{n} x_{n}}{Q_{n}}$

is called the Riesz mean $\left(T, q_{n}\right)$ or simply the $\left(T, q_{n}\right)$ means of a sequence $\left(x_{n}\right)$, where $\left(q_{k}\right)$ is a sequence of positive numbers and $Q_{n}=q_{0}+q_{1}+\cdots+q_{n}$.

The matrix of the $\left(T, q_{n}\right)$ method is given by

$t_{n k}^{q}:=\left\{\begin{array}{cll}\frac{q_{k}}{Q_{n}} & , \quad(0 \leq k \leq n), \\ 0 & , \quad(k>n) .\end{array}\right.$

In order to avoid any confusion throughout the article the symbol $\left(R, q_{n}\right)$ will be used instead of Riesz mean $\left(T, q_{n}\right)$. Now, we are ready to establish the set $r^{q}\left(\widetilde{B}_{u}^{p}\right)$, using the Riesz mean and double sequential band matrix.

For $0<p_{k} \leq H<\infty$, let us define the set $r^{q}\left(\widetilde{B}_{u}^{p}\right)$ as the set of all sequences whose $R_{u}^{q} \widetilde{B}$-transforms is in the sequence space $\ell(p)$, that is

$r^{q}\left(\widetilde{B}_{u}^{p}\right)=\left\{x=\left(x_{k}\right) \in w: \sum_{k}\left|\frac{1}{Q_{k}} \sum_{j=0}^{k} u_{j} q_{j} \widetilde{B} x_{j}\right|^{p_{k}}<\infty\right\}$,

where $u=\left(u_{k}\right)$ is an arbitrary fixed sequence.

With the help of the notation of (1), we can rewrite the set $r^{q}\left(\widetilde{B}_{u}^{p}\right)$ by

$r^{q}\left(\widetilde{B}_{u}^{p}\right)=\left\{\ell_{p}\right\}_{R_{u}^{q} \widetilde{B}}$

where $R_{u}^{q} \widetilde{B}=\left(\begin{array}{c}q_{\tilde{B}}^{u} \\ n k\end{array}\right)$ matrix defined as follows:

$r_{n k}^{q_{\tilde{\widetilde{R}}}^{u}}=\left\{\begin{array}{ccc}\frac{1}{Q_{n}}\left(r_{k} u_{k} q_{k}+s_{k} u_{k+1} q_{k+1}\right) & , & 0 \leq k \leq n-1, \\ \frac{r_{n} q_{n} u_{n}}{Q_{n}} & , & k=n, \\ 0 & , & k>n .\end{array}\right.$

Ahead of frequently used sequence $y=\left(y_{k}\right)$ by the $R_{u}^{q} \widetilde{B}$ - transform of any given sequence $x=\left(x_{k}\right)$, i.e.,

$y_{k}=\frac{1}{Q_{k}} \sum_{j=0}^{k} u_{j} q_{j} \widetilde{B} x_{j}$.

From now on when we speak of the sequences $x=\left(x_{k}\right)$ and $y=\left(y_{k}\right)$, we will mean that they are connected with the relation (2).

Now, it is time to give the following theorem.

Theorem 3.1 The set $r^{q}\left(\widetilde{B}_{u}^{p}\right)$ is a linear space together with coordinatewise addition and scalar multiplication, in other words, $r^{q}\left(\widetilde{B}_{u}^{p}\right)$ represents the sequence space.

Proof: Since the proof of this theorem can be obtained by using elementary linear algebra, we omit the details.

Let us return to explaining our main subject. More recently, the Riesz sequence spaces $r^{q}(u, p)$ and $r^{q}\left(\Delta_{u}^{p}\right)$ of non-absolute type have been introduced and studied by Ganie and Sheikh [65, 66]. After then, some new Riesz sequence spaces have been introduced and examined Candan \& Güneş [28] and Candan \& Kılınç [30]. When compared to the corresponding results in the literature; it is seen that the results of the present study are more general and more inclusive.

Now, let us start with one of the main results which is going to be used in later sections. 
Theorem 3.2 Let $0<p_{k} \leq H<\infty$. Then, $r^{q}\left(\widetilde{B}_{u}^{p}\right)$ is the complete linear metric space paronormed by $g$, described via the following equality

$g_{\widetilde{B}}(x)=\left[\sum_{k}\left|\frac{1}{Q_{k}} \sum_{j=0}^{k-1}\left(r_{j} u_{j} q_{j}+s_{j} u_{j+1} q_{j+1}\right) x_{j}+\frac{r_{k} u_{k} q_{k}}{Q_{k}} x_{k}\right|^{p_{k}}\right]^{\frac{1}{M}}$.

Proof: Clearly, in order to prove the theorem, it is sufficient to show that the conditions of the paranorm are satisfied. To do this, firstly we show that the linearity of $r^{q}\left(\widetilde{B}_{u}^{p}\right)$ with respect to the coordinate wise addition and scalar multiplication is true. Let us assume that $z, x \in r^{q}\left(\widetilde{B}_{u}^{p}\right)$. Therefore, the linearity of $r^{q}\left(\widetilde{B}_{u}^{p}\right)$ is obtained from the following rudimentary calculations

$$
\begin{aligned}
g_{\widetilde{B}}(x+z) & =\left[\sum_{k}\left|\frac{1}{Q_{k}} \sum_{j=0}^{k-1}\left(r_{j} u_{j} q_{j}+s_{j} u_{j+1} q_{j+1}\right)\left(x_{j}+z_{j}\right)+\frac{r_{k} u_{k} q_{k}}{Q_{k}}\left(x_{k}+z_{k}\right)\right|^{p_{k}}\right]^{\frac{1}{M}} \\
& \leq\left[\sum_{k}\left|\frac{1}{Q_{k}} \sum_{j=0}^{k-1}\left(r_{j} u_{j} q_{j}+s_{j} u_{j+1} q_{j+1}\right) x_{j}+\frac{r_{k} u_{k} q_{k}}{Q_{k}} x_{k}\right|^{p_{k}}\right]^{\frac{1}{M}} \\
& +\left[\sum_{k}\left|\frac{1}{Q_{k}} \sum_{j=0}^{k-1}\left(r_{j} u_{j} q_{j}+s_{j} u_{j+1} q_{j+1}\right) z_{j}+\frac{r_{k} u_{k} q_{k}}{Q_{k}} z_{k}\right|^{p_{k}}\right]^{\frac{1}{M}} \\
& =g_{\widetilde{B}}(x)+g_{\widetilde{B}}(z)
\end{aligned}
$$

for any $\mu \in \mathbb{R}$ (see $[2$, p. 30])

$|\mu|^{p_{k}} \leq \max \left\{1,|\mu|^{M}\right\}$.

It is fairly easy to get the next two conditions $g_{\widetilde{B}}(\theta)=0$ and $g_{\widetilde{B}}(x)=g_{\widetilde{B}}(-x)$ which are valid for all $x \in r^{q}\left(\widetilde{B}_{u}^{p}\right)$. One more time, the inequalities (3) and (4) result in the subadditivity of $g$ and the following inequality

$g_{\widetilde{B}}(\mu x) \leq \max \left\{1,|\mu|^{M}\right\} g_{\widetilde{B}}(x)$

obviously holds.

Now let us observe that the scalar multiplication is continuous. To do this, let us assume that $\left(x^{n}\right)$ be arbitrary sequence of the points lying in $r^{q}\left(\widetilde{B}_{u}^{p}\right)$ such that $g_{\widetilde{B}}\left(x^{n}-x\right) \rightarrow 0$ and $\left(\mu_{n}\right)$ also be arbitrary sequence of scalars such that $\mu_{n} \rightarrow \mu$. We can get

$$
\begin{aligned}
g_{\widetilde{B}}\left(\mu_{n} x^{n}-\mu x\right) & =\left[\sum_{k}\left|\frac{1}{Q_{k}} \sum_{j=0}^{k-1}\left(r_{j} u_{j} q_{j}+s_{j} u_{j+1} q_{j+1}\right)\left(\mu_{n} x_{j}^{n}-\mu x_{j}\right)\right|^{p_{k}}\right]^{\frac{1}{M}} \\
& \leq\left|\mu_{n}-\mu\right|^{\frac{1}{M}} g_{\widetilde{B}}\left(x^{n}\right)+|\mu|^{\frac{1}{M}} g_{\widetilde{B}}\left(x^{n}-x\right)
\end{aligned}
$$

tending to be zero if we take $n \rightarrow \infty$ since $\left\{g_{\widetilde{B}}\left(x^{n}\right)\right\}$ is bounded due to the inequality

$g_{\widetilde{B}}\left(x^{n}\right) \leq g_{\widetilde{B}}(x)+g_{\widetilde{B}}\left(x^{n}-x\right)$

which is valid because of subadditive of $g_{\widetilde{B}}$. This shows that the scalar multiplication is continuous. Namely, from now on, we say that $g_{\widetilde{B}}$ is paranorm on the space $r^{q}\left(\widetilde{B}_{u}^{p}\right)$.

Here, if we prove the completeness of the space $r^{q}\left(\widetilde{B}_{u}^{p}\right)$ then the proof ends. Let us suppose that $\left\{x^{i}\right\}$ be an arbitrary Cauchy sequence in the space $r^{q}\left(\widetilde{B}_{u}^{p}\right)$, where $x^{i}=\left\{x_{0}^{i}, x_{1}^{i}, \ldots\right\}$. In that case, there exists a positive integer $n_{0}(\epsilon)$

$g_{\widetilde{B}}\left(x^{i}-x^{j}\right)<\infty$

for all $i, j \geq n_{0}(\epsilon)$ for a given $\epsilon>0$. We get by using definition of $g_{\widetilde{B}}$, for each fixed $k \in \mathbb{N}$

$$
\left|\left(R_{u}^{q} \widetilde{B} x^{i}\right)_{k}-\left(R_{u}^{q} \widetilde{B} x^{j}\right)_{k}\right| \leq\left[\sum_{k}\left|\left(R_{u}^{q} \widetilde{B} x^{i}\right)_{k}-\left(R_{u}^{q} \widetilde{B} x^{j}\right)_{k}\right|^{p_{k}}\right]^{\frac{1}{M}}<\infty
$$


for $i, j \geq n_{0}(\epsilon)$. This result in the fact that $\left\{\left(R_{u}^{q} \widetilde{B} x^{0}\right)_{k},\left(R_{u}^{q} \widetilde{B} x^{1}\right)_{k}, \ldots\right\}$ is a Cauchy sequence of real number for every fixed $k \in \mathbb{N}$. Since we already know that $\mathbb{R}$ is complete, it converge, say $\left(R_{u}^{q} \widetilde{B} x^{i}\right)_{k} \rightarrow\left(R_{u}^{q} \widetilde{B} x\right)_{k}$ as $i \rightarrow \infty$. Using these infinitely many limits $\left(R_{u}^{q} \widetilde{B} x\right)_{0},\left(R_{u}^{q} \widetilde{B} x\right)_{1}, \ldots$ we describe the sequence $\left\{\left(R_{u}^{q} \widetilde{B} x\right)_{0},\left(R_{u}^{q} \widetilde{B} x\right)_{1}, \ldots\right\}$. From (5) for each $m \in \mathbb{N}$ and $i, j \geq n_{0}(\epsilon)$, we have

$\sum_{k=0}^{m}\left|\left(R_{u}^{q} \widetilde{B} x^{i}\right)_{k}-\left(R_{u}^{q} \widetilde{B} x^{j}\right)_{k}\right|^{p_{k}} \leq g_{\widetilde{B}}\left(x^{i}-x^{j}\right)^{M}<\epsilon^{M}$.

Let us pass to limit first as $j \rightarrow \infty$ and next as $m \rightarrow \infty$ in (6), then we obtain $g_{\widetilde{B}}\left(x^{i}-x\right) \leq \infty$

Finally, if we take $\epsilon=1$ in (6) and $i \geq n_{0}(1)$, then using Minkowsky's inequality for each $m \in \mathbb{N}$, we have

$$
\left[\sum_{k=0}^{m}\left|\left(R_{u}^{q} \widetilde{B} x\right)_{k}\right|^{p_{k}}\right]^{\frac{1}{M}} \leq g_{\widetilde{B}}\left(x^{i}-x\right)+g_{\widetilde{B}}\left(x^{i}\right) \leq 1+g_{\widetilde{B}}\left(x^{i}\right) .
$$

This imply that $x \in r^{q}\left(\widetilde{B}_{u}^{p}\right)$. Because of the fact that $g_{\widetilde{B}}\left(x^{i}-x\right) \leq \infty$ for all $i \geq n_{0}(\epsilon)$, it is reached the end of that $x^{i} \rightarrow x$ as $i \rightarrow \infty$, thus we show that $r^{q}\left(\widetilde{B}_{u}^{p}\right)$ is complete and proof of the theorem is completed.

Note that, it can easily be seen that the absolute property is invalid on the space $r^{q}\left(\widetilde{B}_{u}^{p}\right)$, that is $g_{\widetilde{B}}(x) \neq g_{\widetilde{B}}(|x|)$ for at least one sequence in the space $r^{q}\left(\widetilde{B}_{u}^{p}\right)$ and this says us that $r^{q}\left(\widetilde{B}_{u}^{p}\right)$ is a sequence space of non-absolute type.

Theorem 3.3 Let $0<p_{k} \leq H<\infty$. Then the sequence space $r^{q}\left(\widetilde{B}_{u}^{p}\right)$ is linearly isomorphic to the space $\ell(p)$.

Proof: The first step in proving the theorem is to show the existence of a linear bijection between the spaces $r^{q}\left(\widetilde{B}_{u}^{p}\right)$ and $\ell(p)$, where $0<p_{k} \leq H<\infty$. For this, we use the notation of $(3)$, consider the transformation $\mathrm{T}$ that defined from $r^{q}\left(\widetilde{B}_{u}^{p}\right)$ to $\ell(p)$ by $x \rightarrow y=T x$. Indeed, the linearity of $\mathrm{T}$ is fairly easy. Also it is clear that $x=\theta$ whenever $T x=\theta$. Hence $\mathrm{T}$ is injective.

Let $y \in \ell(p)$ and define the sequence $x=\left(x_{k}\right)$ as follows:

$x_{k}=\sum_{n=0}^{k-1} \prod_{j=n+1}^{k}\left(\frac{-s_{j}}{r_{j+1}}\right)\left(\frac{1}{r_{n} u_{n} q_{n}}+\frac{1}{s_{n} u_{n+1} q_{n+1}}\right) Q_{n} y_{n}+\frac{Q_{k}}{r_{k} u_{k} q_{k}} y_{k}$

for $k \in \mathbb{N}$. We eventually obtain,

$$
\begin{aligned}
g_{\widetilde{B}}(x) & =\left[\sum_{k}\left|\frac{1}{Q_{k}} \sum_{j=0}^{k-1}\left(r_{j} u_{j} q_{j}+s_{j} u_{j+1} q_{j+1}\right) x_{j}+\frac{r_{k} u_{k} q_{k}}{Q_{k}} x_{k}\right|^{p_{k}}\right]^{\frac{1}{M}} \\
& =\left[\sum_{k} \mid \sum_{j=0}^{k} \delta_{k j} y_{j}^{p_{k}}\right]^{\frac{1}{M}} \\
& =\left[\sum_{k}\left|y_{k}\right|^{p_{k}}\right]^{\frac{1}{M}} \\
& =g_{1}(y)<\infty
\end{aligned}
$$

where

$\delta_{k j}= \begin{cases}1, & k=j \\ 0, & k \neq j\end{cases}$

Consequently, we obtain that $x \in r^{q}\left(\widetilde{B}_{u}^{p}\right)$ i.e., $\mathrm{T}$ is surjective and its paranorm is preserving. Hence $\mathrm{T}$ is a linear bijection and the proof is completed. 


\section{Basis and $\alpha-, \beta-$ and $\gamma-$ duals of the space $r^{q}\left(\widetilde{B}_{u}^{p}\right)$}

In this section, after we define $M(\lambda, \mu)$ multiplier space of any sequence spaces $\lambda$ and $\mu$, we will research basis and $\alpha-, \beta-$ and $\gamma-$ duals of the space $r^{q}\left(\widetilde{B}_{u}^{p}\right)$.

If $\lambda, \mu \subset w$ and $z$ arbitrary sequence, we can write

$z^{-1} * \lambda=\left\{x=\left(x_{k}\right) \in w: x z=\left(x_{k} z_{k}\right) \in \lambda\right\}$

and

$M(\lambda, \mu)=\cap_{x \in \lambda} x^{-1} * \mu$.

If we choose $\mu=\ell_{1}, c s$ and $b s$, then we obtain the $\alpha-, \beta$ - and $\gamma$ - duals of the space $\lambda$, respectively as

$\lambda^{\alpha}=M\left(\lambda, \ell_{1}\right)=\left\{a=\left(a_{k}\right) \in w: a x=\left(a_{k} x_{k}\right) \in \ell_{1}\right.$ for all $\left.x \in \lambda\right\}$,

$\lambda^{\beta}=M(\lambda, c s)=\left\{a=\left(a_{k}\right) \in w: a x=\left(a_{k} x_{k}\right) \in c s\right.$ for all $\left.x \in \lambda\right\}$,

$\lambda^{\gamma}=M(\lambda, b s)=\left\{a=\left(a_{k}\right) \in w: a x=\left(a_{k} x_{k}\right) \in b s\right.$ for all $\left.x \in \lambda\right\}$.

Let us now state the following lemmas. In this way, the fundamental results will be used in proofs of our theorems.

Lemma $4.1[67]$

(i) Let $1<p_{k} \leq H<\infty$. Then $A \in\left(\ell(p): \ell_{1}\right)$ if and only if there exists an integer $B>1$ such that

$$
\sup _{K \in F} \sum_{k}\left|\sum_{n \in K} a_{n k} B^{-1}\right|^{p_{k}^{\prime}}<\infty
$$

(ii) Let $0<p_{k} \leq 1$. Then $A \in\left(\ell(p): \ell_{1}\right)$ if and only if

$$
\sup _{K \in F} \sup _{k}\left|\sum_{n \in K} a_{n k}\right|^{p_{k}}<\infty \text {. }
$$

Lemma $4.2[68]$

(i) Let $1<p_{k} \leq H<\infty$. Then $A \in\left(\ell(p): \ell_{\infty}\right)$ if and only if there exists an integer $B>1$ such that

$\sup _{n} \sum_{k}\left|a_{n k} B^{-1}\right|^{p_{k}^{\prime}}<\infty$

(ii) Let $0<p_{k} \leq 1$ for every $k \in \mathbb{N}$. Then $A \in\left(\ell(p): \ell_{\infty}\right)$ if and only if

$\sup _{n, k}\left|a_{n k}\right|^{p_{k}}<\infty$.

Lemma $4.3[68] A \in(\ell(p): c)$ if and only if there exists an integer $B>1$ provided that (7) and (8) hold,

$\lim _{n} a_{n k}=\beta_{k}$ for $k \in \mathbb{N}$

also holds. Where $0<p_{k} \leq H<\infty$ for every given $k \in \mathbb{N}$.

Theorem 4.4 Let $0<p_{k} \leq 1$ for all $k \in \mathbb{N}$ and the sets $D_{1}(u, p), D_{2}(u, p)$ and $D_{3}(u, p)$ are defined by following equations

$D_{1}(u, p)=\bigcup_{B>1}\left\{a=\left(a_{k}\right) \in w: \sup _{K \in F} \sum_{k}\left|\sum_{n \in K}\left[\prod_{j=n}^{k-1}\left(\frac{-s_{j}}{r_{j+1}}\right) A_{k} a_{n} Q_{k}+\frac{a_{n}}{r_{n} u_{n} q_{n}} Q_{n}\right] B^{-1}\right|^{p_{k}^{\prime}}<\infty\right\}$, 
$D_{2}(u, p)=\bigcup_{B>1}\left\{a=\left(a_{k}\right) \in w: \sup _{n} \sum_{k}\left|\left[\left(\frac{a_{k}}{r_{k} u_{k} q_{k}}+A_{k} \sum_{i=k+1}^{n} a_{i} \prod_{j=k+1}^{i}\left(\frac{-s_{j-1}}{r_{j}}\right)\right) Q_{k}\right] B^{-1}\right|^{p_{k}^{\prime}}<\infty\right\}$,

where $A_{k}=A\left(r_{k}, s_{k}, u_{k}, q_{k}\right)=\frac{1}{r_{k} u_{k} q_{k}}+\frac{1}{s_{k} u_{k+1} q_{k+1}}$, and

$D_{3}(u, p)=\left\{a=\left(a_{k}\right) \in w: \lim _{n \rightarrow \infty} \sum_{i=k+1}^{n} a_{i} \prod_{j=k+1}^{i}\left(\frac{-s_{j-1}}{r_{j}}\right)\right.$ exists $\}$.

In this case,

$\left[r^{q}\left(\widetilde{B}_{u}^{p}\right)\right]^{\alpha}=D_{1}(u, p), \quad\left[r^{q}\left(\widetilde{B}_{u}^{p}\right)\right]^{\beta}=D_{2}(u, p) \cap D_{3}(u, p), \quad\left[r^{q}\left(\widetilde{B}_{u}^{p}\right)\right]^{\gamma}=D_{2}(u, p)$.

Proof: Firstly, let research that $\alpha$ - dual of space $r^{q}\left(\widetilde{B}_{u}^{p}\right)$. Hence we will consider definition of $\alpha-$ dual. Let us suppose that any $a=\left(a_{k}\right) \in w$. Then we easily obtain by means of that

$\begin{aligned} a_{n} x_{n} & =\sum_{k=0}^{n-1} \prod_{j=k}^{n-1}\left(\frac{-s_{j-1}}{r_{j}}\right)\left(\frac{1}{r_{k} u_{k} q_{k}}+\frac{1}{s_{k} u_{k+1} q_{k+1}}\right) a_{n} Q_{k} y_{k}+\frac{a_{n}}{r_{n} u_{n} q_{n}} Q_{n} y_{n} \\ & =(D y)_{n}\end{aligned}$

where the matrix $D=\left(d_{n k}\right)$ is defined by

$d_{n k}=\left\{\begin{array}{ccc}\prod_{j=n}^{k}\left(\frac{-s_{j-1}}{r_{j}}\right)\left(\frac{1}{r_{k} u_{k} q_{k}}+\frac{1}{s_{k} u_{k+1} q_{k+1}}\right) a_{n} Q_{n} & , 0 \leq k \leq n-1, \\ \frac{a_{n}}{r_{n} u_{n} q_{n}} Q_{n} & , & k=n, \\ 0 & , & k>n,\end{array}\right.$

for all $n, k \in \mathbb{N}$. Thus we observe from (10) that $a x=\left(a_{n} x_{n}\right) \in \ell_{1}$ whenever $x=\left(x_{n}\right) \in r^{q}\left(\widetilde{B}_{u}^{p}\right)$ if and only if $D y \in \ell_{1}$ whenever $y \in \ell(p)$. This means that $D \in\left(\ell(p), \ell_{1}\right)$. Thus we obtain from Lemma 4.1 (ii) that $\left[r^{q}\left(\widetilde{B}_{u}^{p}\right)\right]^{\alpha}=D_{1}(u, p)$.

Now, let us research that $\beta$ - dual of space $r^{q}\left(\widetilde{B}_{u}^{p}\right)$, using the definition of $\beta$-dual. We consider following equation,

$\begin{aligned} \sum_{k=0}^{n} a_{k} x_{k} & =\sum_{k=0}^{n}\left[\left(\frac{a_{k}}{r_{k} u_{k} q_{k}}+\left(\frac{1}{r_{k} u_{k} q_{k}}+\frac{1}{s_{k} u_{k+1} q_{k+1}}\right) \sum_{i=k+1}^{n} a_{i} \prod_{j=k+1}^{i}\left(\frac{-s_{j-1}}{r_{j}}\right)\right) Q_{k}\right] y_{k} \\ & =(E y)_{n}\end{aligned}$

where, $E=\left(e_{n k}\right)$ is defined as following

$e_{n k}=\left\{\begin{array}{cc}\left(\frac{a_{k}}{r_{k} u_{k} q_{k}}+\left(\frac{1}{r_{k} u_{k} q_{k}}+\frac{1}{s_{k} u_{k+1} q_{k+1}}\right) \sum_{i=k+1}^{n} a_{i} \prod_{j=k+1}^{i}\left(\frac{-s_{j-1}}{r_{j}}\right)\right) Q_{k} & , \quad 0 \leq k \leq n, \\ 0 & , \quad k>n .\end{array}\right.$

From (11), $a x=\left(a_{k} x_{k}\right) \in c s$ whenever $x \in r^{q}\left(\widetilde{B}_{u}^{p}\right)$ if and only if $E y \in c$ whenever $y \in \ell(p)$. In other words $E \in(\ell(p), c)$. We obtain $\left[r^{q}\left(\widetilde{B}_{u}^{p}\right)\right]^{\beta}=D_{2}(u, p) \cap D_{3}(u, p)$, using Lemma 4.3.

Finally, let find out that $\gamma$ - dual of space $r^{q}\left(\widetilde{B}_{u}^{p}\right)$, using the definition of $\gamma-$ dual. Using (11) $a x=\left(a_{k} x_{k}\right) \in b s$ whenever $x \in r^{q}\left(\widetilde{B}_{u}^{p}\right)$ if and only if $E y \in \ell_{\infty}$ whenever $y \in \ell(p)$. In other words, $a=\left(a_{k}\right) \in\left[r^{q}\left(\widetilde{B}_{u}^{p}\right)\right]^{\gamma}$ iff $E \in\left(\ell(p), \ell_{\infty}\right)$. Then from Lemma 4.2 (ii) obtain $\left[r^{q}\left(\widetilde{B}_{u}^{p}\right)\right]^{\gamma}=D_{2}(u, p)$. Hence, the proof is completed.

Theorem 4.5 Let $1<p_{k} \leq H<\infty$ for every $k \in \mathbb{N}$ and define the sets $D_{4}(u, p)$ and $D_{5}(u, p)$ with the following equations

$D_{4}(u, p)=\left\{a=\left(a_{k}\right) \in w: \sup _{K \in F} \sup _{k}\left|\sum_{n \in K}\left[\prod_{j=n+1}^{k}\left(\frac{-s_{j-1}}{r_{j}}\right)\left(\frac{1}{r_{k} u_{k} q_{k}}+\frac{1}{s_{k} u_{k+1} q_{k+1}}\right) a_{n} Q_{k}+\frac{a_{n}}{r_{n} u_{n} q_{n}} Q_{n}\right]\right|^{p_{k}}<\infty\right\}$, 
$D_{5}(u, p)=\left\{a=\left(a_{k}\right) \in w: \sup _{k}\left|\left[\left(\frac{a_{k}}{r_{k} u_{k} q_{k}}+\left(\frac{1}{r_{k} u_{k} q_{k}}+\frac{1}{s_{k} u_{k+1} q_{k+1}}\right) \sum_{i=k+1}^{n} a_{i} \prod_{j=k+1}^{i}\left(\frac{-s_{j-1}}{r_{j}}\right)\right) Q_{k}\right]\right|^{p_{k}}<\infty\right\}$.

Then,

$\left[r^{q}\left(\widetilde{B}_{u}^{p}\right)\right]^{\alpha}=D_{4}(u, p), \quad\left[r^{q}\left(\widetilde{B}_{u}^{p}\right)\right]^{\beta}=D_{3}(u, p) \cap D_{5}(u, p), \quad\left[r^{q}\left(\widetilde{B}_{u}^{p}\right)\right]^{\gamma}=D_{5}(u, p)$.

Proof: The proof of theorem is obtained alike the proof of Theorem 4.4.

Theorem 4.6 Let $0<p_{k} \leq H<\infty$ for all $k \in \mathbb{N}$. Define the sequence $b^{(k)}(q)=\left\{b_{n}^{(k)}(q)\right\}$ of the elements of the space $r^{q}\left(\widetilde{B}_{u}^{p}\right)$ for every fixed $k \in \mathbb{N}$ by

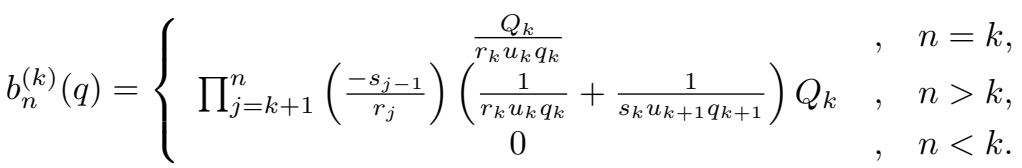

Then, the sequence $b^{(k)}(q)$ is a basis for the space $r^{q}\left(\widetilde{B}_{u}^{p}\right)$ and any $x \in r^{q}\left(\widetilde{B}_{u}^{p}\right)$ has a unique representation of the form

$x=\sum_{k} \lambda_{k}(q) b^{k}(q)$

where, $\lambda_{k}(q)=\left(R_{u}^{q} \widetilde{B} x\right)_{k}$ for all $k \in \mathbb{N}$.

Proof: Let $0<p_{k} \leq H<\infty$. Then it is not difficult to verify of the relation $\left\{b^{(k)}(q)\right\} \subset r^{q}\left(\widetilde{B}_{u}^{p}\right)$. Really, for $k \in \mathbb{N}$

$R_{u}^{q} \widetilde{B} b^{(k)}(q)=e^{(k)} \in \ell(p)$

where $e^{(k)}$ is a sequence that 1 in $k^{t h}$ term for each $k \in \mathbb{N}$ another term 0 .

Moreover, let $x \in r^{q}\left(\widetilde{B}_{u}^{p}\right)$. For all non-negative integer $m$, we put

$x^{[m]}=\sum_{k=0}^{m} \lambda_{k}(q) b^{(k)}(q)$.

In this case, we have that $R_{u}^{q} \widetilde{B}$ to (14) that for $i, m \in \mathbb{N}$

$R_{u}^{q} \widetilde{B} x^{[m]}=\sum_{k=0}^{m} \lambda_{k}(q) R_{u}^{q} \widetilde{B} b^{(k)}(q)=\sum_{k=0}^{m}\left(R_{u}^{q} \widetilde{B} x\right)_{k} e^{(k)}$

and hence

$\left(R_{u}^{q} \widetilde{B} x-x^{[m]}\right)_{i}=\left\{\begin{array}{cc}0 & 0 \leq i \leq m, \\ \left(R_{u}^{q} \widetilde{B} x\right)_{i} & , \quad i>m .\end{array}\right.$

Also, for any given $\epsilon>0$, there exists an integer $m_{0}$ such that for every $m \geq m_{0}$

$\left(\sum_{i=m_{0}}^{\infty}\left|\left(R_{u}^{q} \widetilde{B} x\right)_{i}\right|^{p_{k}}\right)^{\frac{1}{M}}<\frac{\epsilon}{2}$

Hence, we obtain for all $m \geq m_{0}$ that

$$
\begin{aligned}
g_{\widetilde{B}}\left(x-x^{m}\right) & =\left(\sum_{i=m}^{\infty}\left|\left(R_{u}^{q} \widetilde{B} x\right)_{i}\right|^{p_{k}}\right)^{\frac{1}{M}} \\
& \leq\left(\sum_{i=m_{0}}^{\infty}\left|\left(R_{u}^{q} \widetilde{B} x\right)_{i}\right|^{p_{k}}\right)^{\frac{1}{M}} \\
& <\frac{\epsilon}{2}<\epsilon .
\end{aligned}
$$


A little rewriting and the use of limit properties give $\lim _{m \rightarrow \infty} g_{\widetilde{B}}\left(x-x^{m}\right)=0$ that is $x$ is represented as (12).

Now, if we prove the uniqueness of the representation (12) of $x \in r^{q}\left(\widetilde{B}_{u}^{p}\right)$ then the proof ends. For this, suppose the contrary. That is, it have two representation like $x=\sum_{k} \mu_{k}(q) b^{(k)}$ and $x=\sum_{k} \lambda_{k}(q) b^{(k)}$.

Since we know that the linear transformation from $r^{q}\left(\widetilde{B}_{u}^{p}\right)$ to $\ell(p)$ is continuous, we get

$$
\begin{aligned}
\left(R_{u}^{q} \widetilde{B} x\right)_{n} & =\sum_{k} \mu_{k}(q)\left(R_{u}^{q} \widetilde{B} b^{(k)}(q)\right)_{n} \\
& =\sum_{k} \mu_{k}(q) e_{n}^{(k)}=\mu_{n}(q)
\end{aligned}
$$

for $n \in \mathbb{N}$. We acknowledge that $\left(R_{u}^{q} \widetilde{B} x\right)_{n}=\lambda_{n}$ for all $n \in \mathbb{N}$. Hence $\lambda_{n}(q)=\mu_{n}(q)$ and so unique of representation (12) is obtained. This ends the proof of the last part of the theorem.

\section{Matrix Mapping on the Space $r^{q}\left(\widetilde{B}_{u}^{p}\right)$}

One of the most important ideas is matrix transformation in this article. So, we focus on this concept in the present section. Now, we emphasize the characterization of $\left(r^{q}\left(\widetilde{B}_{u}^{p}\right), \ell_{\infty}\right)$.

Theorem 5.1 (i) $A \in\left(r^{q}\left(\widetilde{B}_{u}^{p}\right), \ell_{\infty}\right)$ if and only if there exists an integer $B>0$ such that

$C(B)=\sup _{n} \sum_{k}\left|\left[\left(\frac{a_{n k}}{r_{k} u_{k} q_{k}}\right)+\left(\frac{1}{r_{k} u_{k} q_{k}}+\frac{1}{s_{k} u_{k+1} q_{k+1}}\right) \sum_{i=k+1}^{n} a_{n i} \prod_{j=k+1}^{i}\left(\frac{-s_{j-1}}{r_{j}}\right)\right] Q_{k} B^{-1}\right|^{p_{k}^{\prime}}<\infty$

and

$\left\{a_{n k}\right\}_{k \in \mathbb{N}} \in c s \quad(n \in \mathbb{N})$

where $1<p_{k} \leq H<\infty$ for every $k \in \mathbb{N}$.

(ii) $A \in\left(r^{q}\left(\widetilde{B}_{u}^{p}\right), \ell_{\infty}\right)$ if and only if

$\sup _{n, k}\left|\left[\left(\frac{a_{n k}}{r_{k} u_{k} q_{k}}\right)+\left(\frac{1}{r_{k} u_{k} q_{k}}+\frac{1}{s_{k} u_{k+1} q_{k+1}}\right) \sum_{i=k+1}^{n} a_{n i} \prod_{j=k+1}^{i}\left(\frac{-s_{j-1}}{r_{j}}\right)\right] Q_{k}\right|^{p_{k}}<\infty$

and

$\left\{a_{n k}\right\}_{k \in \mathbb{N}} \in c s \quad(n \in \mathbb{N})$

where $0<p_{k} \leq 1<\infty$ for every $k \in \mathbb{N}$.

Proof: (i) Let $1<p_{k} \leq H<\infty$ for every $k \in \mathbb{N}$ and $A \in\left(r^{q}\left(\widetilde{B}_{u}^{p}\right), \ell_{\infty}\right)$. Then $A x$ exists for $x \in$ $r^{q}\left(\widetilde{B}_{u}^{p}\right), \quad\left\{a_{n k}\right\}_{k \in \mathbb{N}} \in\left[r^{q}\left(\widetilde{B}_{u}^{p}\right)\right]^{\beta}$ for each $n \in \mathbb{N}$. Also consider the following equality obtained by using the relation (10) that

$\sum_{k=0}^{m} a_{n k} x_{k}=\sum_{k=0}^{m}\left[\left(\frac{a_{n k}}{r_{k} u_{k} q_{k}}+\left(\frac{1}{r_{k} u_{k} q_{k}}+\frac{1}{s_{k} u_{k+1} q_{k+1}}\right) \sum_{i=k+1}^{n} a_{n i} \prod_{j=k+1}^{i}\left(\frac{-s_{j-1}}{r_{j}}\right)\right) Q_{k}\right] y_{k}$.

From Lemma (4.1) and (17), we obtain (15) expression.

Conversely, supposed that is provided (15) expression and $\left\{a_{n k}\right\}_{k \in \mathbb{N}} \in c s$ for each $n \in \mathbb{N} \quad x \in r^{q}\left(\widetilde{B}_{u}^{p}\right)$. Since $\left\{a_{n k}\right\}_{k \in \mathbb{N}} \in\left[r^{q}\left(\widetilde{B}_{u}^{p}\right)\right]^{\beta}$ for every fixed $n \in \mathbb{N}$, A-transform of $x$ exists. We easily can derive from $(17)$ as $m \rightarrow \infty$ that

$\sum_{k=0}^{\infty} a_{n k} x_{k}=\sum_{k=0}^{\infty}\left[\left(\frac{a_{n k}}{r_{k} u_{k} q_{k}}+\left(\frac{1}{r_{k} u_{k} q_{k}}+\frac{1}{s_{k} u_{k+1} q_{k+1}}\right) \sum_{i=k+1}^{\infty} a_{i k} \prod_{j=k+1}^{i}\left(\frac{-s_{j-1}}{r_{j}}\right)\right) Q_{k}\right] y_{k}$. 
Now, by combining (18) and inequality holding for an arbitrary $Z>0$ and complex numbers $a, b$

$|a b| \leq Z\left\{\left|a Z^{-1}\right|^{p^{\prime}}+|b|^{p}\right\}$

where $p>1$ and $1 / p+1 / p^{\prime}=1$. We can easily obtain

$$
\begin{aligned}
\sup _{n \in \mathbb{N}}\left|\sum_{k=0}^{\infty} a_{n k} x_{k}\right| & \leq \sup _{n \in \mathbb{N}} \sum_{k=0}^{\infty}\left|\left[\left(\frac{a_{n k}}{r_{k} u_{k} q_{k}}+\left(\frac{1}{r_{k} u_{k} q_{k}}+\frac{1}{s_{k} u_{k+1} q_{k+1}}\right) \sum_{i=k+1}^{\infty} a_{i k} \prod_{j=k+1}^{i}\left(\frac{-s_{j-1}}{r_{j}}\right)\right) Q_{k}\right]\right|\left|y_{k}\right| \\
& \leq Z\left[C(B)+g_{1}^{M}(y)\right]<\infty .
\end{aligned}
$$

This mean that $A x \in \ell_{\infty}$ whenever $x \in r^{q}\left(\widetilde{B}_{u}^{p}\right)$. Hence, the proof is completed. (ii) The proof of (ii) be done alike (i).

\section{Note}

Some results of this work were partially presented at the $4^{\text {th }}$ International Eurasian Conference on Mathematical Sciences and Applications (IECMSA 2015) to be held on 31 August-03 September 2015 in Athens, GREECE.

\section{References}

[1] F. Başar, Summability Theory and Its Applications, Bentham Science Publishers, e-books, Monographs, xi+405 pp., İstanbul 2012, ISB:978-1-60805-252-3.

[2] I. J. Maddox, Paranormed sequence spaces generated by infinite matrices, Proc. Cambridge. Philos. Soc. 64(1968), $335-340$.

[3] I. J. Maddox, Spaces of strongly summable sequences, Quart. J. Math. Oxford, 18(2)(1967), 345-355.

[4] S. Simons, The sequence spaces $\ell\left(p_{v}\right)$ and $m\left(p_{v}\right)$, Proc. London Math. Soc., 15(3)(1965), 422-436.

[5] H. Nakano, Modulared sequence spaces, Proc. Japan Acad. 27(2)(1951), 508-512.

[6] P.N. Ng and P.Y. Lee, Cesro sequence spaces of non-absolute type, Comment. Math. Prace Mat. 20(1978), no.2, 429-433.

[7] B. Altay and F. Başar, On the paranormed Riesz sequence spaces of non-absolute type, Southeast Asian Bull. Math. 26(2002), 701-715.

[8] B. Altay, F. Başar, M. Mursaleen, On the Euler sequence spaces which include the spaces $\ell_{p}$ and $\ell_{\infty} I$, Inform. Sci. 176(10)(2006), 1450-1462.

[9] M. Mursaleen, F. Başar, B. Altay, On the Euler sequence spaces which include the spaces $\ell_{p}$ and $\ell_{\infty} I I$, Nonlinear Anal. 65(3)(2006), 707-717.

[10] E.E. Kara, M. Öztürk, M. Başarır, Some topological and geometric properties of generalized Euler sequence spaces, Math. Slovaca 60(3)(2010), 385-398.

[11] E. Malkowsky and E. Savaş, Matrix transformations between sequence spaces of generalized weighted means, Appl. Math. Comput. 147(2004), 333-345.

[12] B. Altay, F. Başar, Generalization of the sequence space $\ell(p)$ derived by weighted mean, J. Math. Anal. Appl. 330(2007), 174-185.

[13] C. Aydın and F. Başar, Some new sequence spaces which include the spaces $\ell_{p}$ and $\ell_{\infty}$, Demonstratio Math. 38(3)(2005), 641-656.

[14] C. Aydın and F. Başar, Some generalizations of the sequence spaces $a_{p}^{r}$, Iran. J. Sci. Technol. Trans.A.Sci. 30(A2)(2006), 175-190.

[15] E. Malkowsky, V. Rakočević, S. Źivković, Matrix transformations between the sequence space bv ${ }^{p}$ and certain BK spaces, Bull. Cl. Sci. Math. Nat. Sci. Math. 27(2002), 33-46. 
[16] F. Başar and B. Altay, On the space of sequences of p-bounded variation and related matrix mappings, Ukranian Math. J. 55(2003), 136-147.

[17] B. Altay and F. Başar, Some paranormed sequence spaces of non-absolute type derived by weighted mean, J. Math. Anal. Appl. 319(2)(2006), 494-508.

[18] B. Choudhary and S. K. Mishra, On Köthe-Toeplitz duals of certain sequence spaces and their matrix transformations, Indian J. Pure Appl. Math. 24(1993), no.5, 291-301.

[19] M. Mursaleen, A.K. Noman, On some new sequence spaces of non-absolute type related to the spaces $\ell_{p}$ and $\ell_{1} I$, Filomat $\mathbf{2 5}(2)(2011), 33-51$.

[20] M. Kirişçi, F.Başar, Some new sequence spaces derived by the domain of generalized difference matrix, Comput. Math. Appl. 60(5)(2010), 1299-1309.

[21] M. Candan, Domain of the double sequential band matrix in the classical sequence spaces, J. Inequal. Appl. (2012), 2012:281, 15 pp.

[22] F. Başar, M. Kirişçi, Almost convergence and generalized difference matrix, Comput. Math. Appl. 61(3)(2011), 602-611.

[23] M. Candan, Almost convergence and double sequential band matrix, Acta. Math. Sci. 34B(2)(2014), 354-366.

[24] M. Candan, A new sequence space isomorphic to the space $\ell(p)$ and compact operators J. Math. Comput. Sci. 4 (2014), No. 2, 306-334.

[25] M. Candan, Domain of the double sequential band matrix in the spaces of convergent and null sequences, Adv. Difference Edu.(2014), 2014:163, 18 pp.

[26] M. Candan, Some new sequence spaces derived from the spaces of bounded, convergent and null sequences, Int. J. Mod. Math. Sci.,(12) (2) (2014), 74-87.

[27] M. Candan, A new approach on the spaces of generalized Fibonacci difference null and convergent sequences, Math. AEterna. (1) (5) (2015), 191-210.

[28] M. Candan and A. Güneş, Paranormed sequence space of non-absolute type founded using generalized difference matrix, Proc. Nat. Acad. Sci. India Sect. A., (85) (2) (2015), 269-276.

[29] M. Candan and K. Kayaduman, Almost convergent sequence space derived by generalized Fibonacci matrix and Fibonacci core, Brithish J. Math. Comput. Sci., (7) (2) (2015), 150-167.

[30] M. Candan and G. Kılınç, A different look for paranormed Riesz sequence space of derived by Fibonacci Matrix, Konuralp J. Math. Vol.3, No.2, (2015), 62-76.

[31] M. Candan, E.E. Kara, A study on topological and geometrical characteristics of new Banach sequence spaces, Gulf J. Math. Vol.3, No.4, (2015), 67-84.

[32] M. Kirişçi, On the spaces of Euler almost null and Euler almost convergent sequences, s. Commun. Fac. Sci. Univ. Ank. Ser. A1 Math. Stat. 62(1), 1-16 (2013).

[33] M. Kirişçi, Almost convergence and generalized weighted mean, AIP Conf. Proc., vol. 1470, pp. 191-194 (2012).

[34] M. Kirişçi, Almost Convergence and Generalized Weighted Mean II, J. Inequal. Appl. (2014), 2014:93, 13 pp.

[35] B. Altay and F. Başar, Some paranormed sequence spaces derived by generalized weighted mean, J. Math. Anal. Appl. 319(2006), 494-508.

[36] H. Polat, V. Karakaya, N. Şimşek, Difference sequence spaces derived by generalized weighted mean, Appl. Math. Lett. 24(5)(2011), 608-314.

[37] M. Başarır, On the generalized Riesz B-difference sequence spaces, Filomat 24(4)(2010), 35-52.

[38] B. Altay, F. Başar, On the fine spectrum of the generalized difference operator $B(r, s)$ over the sequence $c_{0}$ and $c$, Int. J. Math. Sci. 18(2008), 3005-3013.

[39] S. Demiriz, C. Çakan, Some topolojical and geometrical properties of a new difference sequence space, Abstr. Appl. Anal. doi:10.1155/2011/213878, 14 pp.

[40] M. Başarır, M. Öztürk, On the Riesz diference sequence space, Rend. Circ. Mat. Palermo 57(2008), 377-389. 
[41] M. Başarır, Paranormed Cesro difference sequence space and related matrix transformation, Doa Tr. J. Math. 15(1991), 14-19.

[42] M. Et, M. Işık, On pa-dual spaces of generalized difference sequence spaces, Appl. Math. Lett. 25(10)(2012), $1486-1489$.

[43] M. Et, Generalized Cesro difference sequence spaces of non-absolute type involving lacunary sequence spaces, Appl. Math. Comput. 219(17)(2013), 9372-9376.

[44] M. Başarır, E.E. Kara, On the $m^{\text {th }}$ order difference sequence space of generalized weighted mean and compact operator, Acta. Math. Sci. 33B(3)(2013), 1-18.

[45] M. Başarır, E.E. Kara, On the B-difference sequence space derived by generalized weighted mean and compact operators, J. Math. Anal. Appl. 391(2012), 67-81.

[46] M. Başarır, M. Kayıkçı, On the generalized $B^{\text {th }}$-Riesz difference sequence space and beta-property J. Inequal. Appl. (2009), ID 385029, 18pp.

[47] M. Başarır, E.E. Kara, On some difference sequence spaces of weighted means and compact operators, Ann. Funct. Anal. $\mathbf{2}(2)(2011), 116-131$.

[48] M. Başarır, E.E. Kara, On compact operators on the Riesz $B^{m}$-difference sequence space, Iran. J. Sci. Technol. Trans. 35A(4)(2011), 279-285.

[49] M. Başarır, E.E. Kara, On compact operators on the Riesz $B^{m}$-difference sequence space-II, Iran. J. Sci. Technol. Trans. 36A(3)(2012), 371-376.

[50] M. Başarır, M. Öztürk, On some Generalized $B^{m}$-difference Riesz Sequence Spaces and Uniform Opial Property, J. Inequal. Appl. (2011), 2011:485730, 17 pp.

[51] Ş. Konca, M. Başarır, Generalized difference sequence spaces associated with a multiplier sequence on a real $n-$ normed space, J. Inequal. Appl. (2013), 2013:335, 12 pp.

[52] Ş. Konca, M. Başarır, On some spaces of almost lacunary convergent sequences derived by Riesz mean and weighted almost lacunary statistical convergence in a real normedspace, J. Inequal. Appl. (2014), 2013:81, 11 pp.

[53] M. Candan, İ. Solak, On some Difference Sequence Spaces Generated by Infinite Matrices, International Journal of Pure and Applied Mathematics, V.25(1), 79-85, 2005.

[54] M. Candan, İ. Solak, On New Difference Sequence Spaces Generated by Infinite Matrices, International Journal of Science and Tecnology, V 1(1), 15-17, 2006.

[55] M. Candan, Some new sequence spaces defined by a modulus function and an infinite matrix in a seminormed space, J. Math. Anal., 3(2)(2012), 1-9.

[56] Y. Yılmaz, M.K. Özdemir, İ. Solak, M. Candan, Operators on some vector-valued Orlicz sequence spaces, F.. Fen ve Mhendislik Dergisi, 17(1), 59-71, (2005).

[57] H. Kizmaz, On certain sequence spaces, Canad. Math. Bull. 24(2)(1981), 169-176.

[58] B. Altay, F. Başar, The matrix domain and the fine spectrum of the difference operator $\Delta$ on the sequence space $\ell_{p}$, $(0<p<1)$, Commun. Math. Anal. 2(2)(2007), 1-11.

[59] F. Başar, B. Altay, On the space of sequences of p-bounded variation and related matrix mappings, Ukrainian Math. J. 55(1)(2003), 136-147.

[60] R. Çolak, M. Et, E. Malkowsky, Some Topics of Sequence Spaces, Lecture Notes in Mathematics, Firat Univ. Elâzı̆̆, Turkey, 2004, pp. 1-63, Frrat Univ. Press, 2004, ISBN: 975-394-038-6.

[61] R. Çolak, M. Et, On some generalized difference sequence spaces and related matrix transformations, Hokkaido Math. J., 26(3) (1997), 483-492.

[62] E. Malkowsky, S.D. Parashar, Matrix transformations in space of bounded and convergent difference sequence of order $m$, Analysis 17(1997), 87-97.

[63] H. Polat, F. Başar, Some Euler spaces of difference sequences of order m, Acta Math. Sci. 27B(2)(2007), 254-266.

[64] B. Altay, On the space of $p$-summable difference sequences of order $m,(1 \leq p<\infty)$, Stud. Sci. Math. Hungar. 43(4)(2006), 387-402. 
[65] N.A. Sheikh, A.H. Ganie, A new paranormed sequence space and some matrix transformations, Acta Math. Acad. Paedago. Nyregy., 28(2012), 47-58.

[66] A.H. Ganie, N.A. Sheikh, New type of paranormed sequence space of non-absolute type and a matrix transformation, Int. J. of Mod. Math. Sci, 8 (2) (2013), 196-211.

[67] K. -G. Grosse-Erdmann, Matrix transformations between the sequence spaces of Maddox, J. Math. Anal. Appl. 180(1993), 223-238.

[68] C.G. Lascarides, I.J. Maddox, Matrix transformations between some classes of sequences, Proc. Cambridge Philos.Soc. 68(1970), 99-104. 